\title{
The Role of Additive Manufacturing in Improving Resource Efficiency and Sustainability
}

\author{
Mélanie Despeisse and Simon Ford \\ Institute for Manufacturing, University of Cambridge, United Kingdom \\ $\{$ ma621, sjf39\}@cam.ac.uk
}

\begin{abstract}
Additive manufacturing is heralded as a revolutionary process technology. While it has yet to cause a dramatic transformation of the manufacturing system, there are early signs of how the characteristics of this novel production process can improve resource efficiency and other sustainability aspects. In this paper, we draw on examples from a wide range of products and industries to understand the role of additive manufacturing in sustainable industrial systems. We identify four main areas in which the adoption of additive manufacturing is leading to improved resource efficiency: (1) product and process design; (2) material input processing; (3) make-to-order product and component manufacturing; and (4) closing the loop.
\end{abstract}

Keywords: Additive manufacturing $\cdot 3 \mathrm{D}$ printing $\cdot$ Sustainability

\section{Introduction}

Advanced manufacturing technologies are leading companies to rethink where and how they manufacture products. Adopting these technologies heralds a future in which value chains are shorter, smaller, more localised and more collaborative, and offers significant sustainability benefits [1]. Such value chain reconfigurations for sustainability will require a better understanding of the relationships and interactions between stakeholders along the product and material life cycles [2] (Fig. 1).

Additive manufacturing (AM) is one of these advanced manufacturing technologies. To date AM and sustainable industrial systems (SIS) have been viewed from different perspectives. In this paper we explore these two topics through a single lens to better understand the implications of AM on the sustainability of industrial systems. More specifically, we address the following question: How can the adoption of additive manufacturing improve the resource efficiency of industrial systems? First we review the characteristics of AM and prior work at the intersection of AM and sustainability. Then we provide examples that led to resource efficiency and other sustainability benefits. These benefits include allowing companies to redesign and simplify components, products and processes for dematerialisation; be more material, energy and cost efficient in various life cycle stages; customise products according to customer preferences; extend product life through repair and remanufacturing; move towards service-based business models; decouple social and economic value creation from environmental impact; and embrace circular economy concepts. 


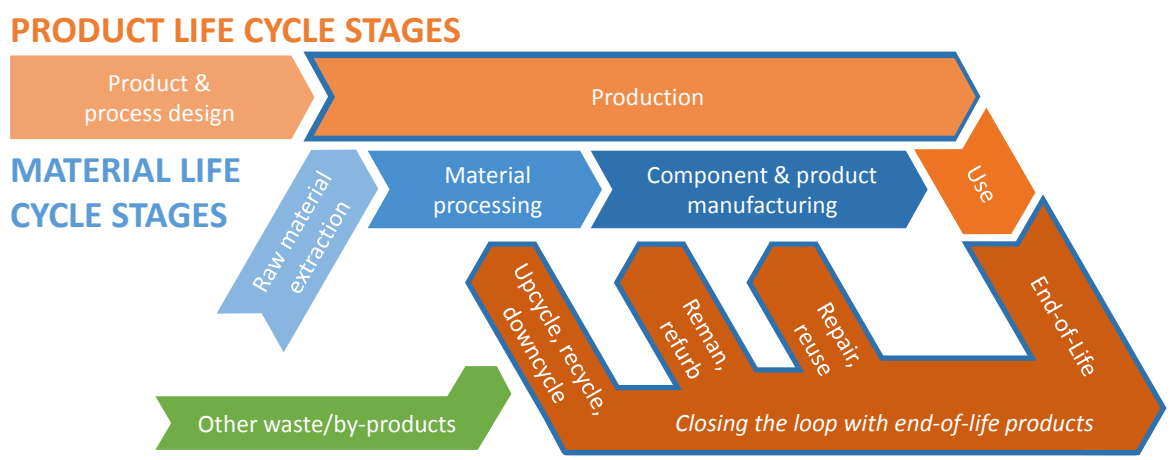

Fig. 1. Product and material life cycle stages

\section{Additive Manufacturing}

AM, also known as 3D printing, allows objects to be fabricated layer by layer, enabling three dimensional objects to be 'printed' on demand [3]. It is gradually being adopted as a direct manufacturing approach in sectors such as aerospace, motorsports, toys, jewellery, along with a number of medical applications where personalisation is key (e.g. hearing aids, orthodontics, prosthetics, implants). These are at various stages of maturity and adoption, and new applications continue to be found as the technology performance improves.

A number of advantages arising from the adoption of AM have been identified $[3,4,5]$. These advantages include the digital nature of the fabrication process. Direct production from 3D CAD models means that no tools and moulds are required, and small batch sizes are more economically attractive relative to traditional manufacturing methods because there are no switch-over costs. Furthermore, these digital files can be easily shared and modified on a distributed basis. Designers also have greater freedoms to create novel structures with AM due to the layer-by-layer deposition of material. The additive nature of the process meaning that less waste material is created and this provides cost savings on material inputs. Direct interaction between the producer and consumers becomes more important through the customisation process. Finally producing goods on demand through AM also reduces inventories and the risks associated with oversupply and obsolescence.

\section{Links between Additive Manufacturing and Sustainability}

As adopting AM technologies will radically transform manufacturing systems, policy makers have begun to consider how they can best support their development and implementation. In the UK, the Additive Manufacturing Special Interest Group of the Materials Knowledge Transfer Network identified a number of potential contributions that AM could make to support future needs in sustainable, high value manufacturing. These included improvements to resource efficiency, more efficient manufacturing systems, 
integrating new materials, implementing new manufacturing processes, and adopting new business models [6].

Life cycle analyses have shown that the adoption of AM could have significant savings in the production and use phases of a product. Estimates for 2025 are in the ranges of \$113-370 billion and \$56-219 billion respectively in each of these phases. The savings in the production phase stems from reduced material inputs and handling, along with shorter supply chains. In the use phase, lightweight components enable energy consumption to be reduced [1].

\subsection{Examples}

There is a growing number of component and product redesign examples [7]. In this section we provide examples in which AM has delivered sustainability benefits at various stages of the product and material life cycles. These are summarised in Table 1.

Table 1. Identified benefits in various life cycle stages for the examples provided

\begin{tabular}{|c|c|c|c|c|c|c|}
\hline & $\begin{array}{l}\text { Product \& pro- } \\
\text { cess design }\end{array}$ & $\begin{array}{l}\text { Material pro- } \\
\text { cessing }\end{array}$ & Manufacturing & $\begin{array}{l}\text { Use \& } \\
\text { service }\end{array}$ & $\begin{array}{l}\text { Repair } \\
\text { \& reman }\end{array}$ & Recycling \\
\hline GE & $\checkmark$ & & $\checkmark$ & $\checkmark$ & & \\
\hline Metalysis & & $\checkmark$ & & & & \\
\hline Bewell Watches & & $\checkmark$ & $\checkmark$ & & & $\checkmark$ \\
\hline Salcomp & $\checkmark$ & & $\checkmark$ & & & \\
\hline Home 3D printers & $\checkmark$ & & $\checkmark$ & $\checkmark$ & & $\checkmark$ \\
\hline 3D Hubs & $\checkmark$ & & $\checkmark$ & $\checkmark$ & & $\checkmark$ \\
\hline Rolls-Royce & & & $\checkmark$ & & $\checkmark$ & \\
\hline PPP & & $\checkmark$ & $\checkmark$ & & & $\checkmark$ \\
\hline Filabot & & $\checkmark$ & & & & $\checkmark$ \\
\hline EKOCYCLE Cube & $\checkmark$ & $\checkmark$ & $\checkmark$ & $\checkmark$ & & $\checkmark$ \\
\hline
\end{tabular}

Product Redesign. Through its collaboration and subsequent acquisition of Morris Technologies, General Electric (GE) developed capabilities in AM. These capabilities have been implemented in the redesign of a fuel nozzle for the LEAP engine that enters production in 2016. The new fuel nozzle is five times stronger to aid its durability. Its design provides the best fuel flow geometry to improve combustion efficiency. GE reduced the nozzle's weight by $25 \%$ through using cobalt chrome and simplifying the design from 20 separate components to a single component [8].

Raw Material Processing. Significant energy is consumed during the refining and processing of metal ores in preparation for manufacturing. The UK-based firm Metalysis has commercialised a process for producing titanium powder directly from titanium ore. This new process requires significantly less energy to produce the titanium powder than the established process [9]. Furthermore, the process uses a non-toxic reactant, calcium chloride $(\mathrm{CaCl})$, during refinement and any leftover $\mathrm{CaCl}$ can be reused. 
Conversion of By-Product into Product. Wood flour and dust are by-products of timber and wood processing. They have traditionally been discarded but have found application in several markets. It is most commonly used as a filler in thermosetting resins, along with wood-plastic composites and building products. Recently, these by-products have been combined with binding agents to create a wood filament that can be used in AM equipment. One company that has taken advantage of this new material input is Bewell Watches, which produces customised wood watches.

Production Process Redesign. To improve the efficiency of power supply casing production, Salcomp aimed to reduce the cooling time in its injection moulding process. Using AM technology, engineers were able to redesign the vent structure of the moulds to dissipate heat more quickly. As a result, cooling time was reduced from 14 to $8 \mathrm{sec}-$ onds, enabling increased production. A secondary benefit was improved quality, with rejection rates reduced from $2.0 \%$ to $1.4 \%$ [10].

Manufacturing System Reconfiguration. The adoption of consumer 3D printers such as the Makerbot Replicator, Ultimaker and Cube are leading to a more distributed and localised manufacturing system. The user becomes both producer and consumer, a prosumer. Individuals with 3D printers are able to design and manufacture the products they require, on-demand and to their own specifications. Logistics are still required for raw material flows but the need for the transport of final products and product inventories is removed. Furthermore, manufacturing locally in the home also makes it possible to create in-situ recycling systems for products made from $3 \mathrm{D}$ printed materials.

Networks such as 3D Hubs provide an online platform that links owners of 3D printers with customers. The owners are typically prosumers who have spare printing capacity and want to increase utilisation. This provides access to local manufacturing. It delivers the same benefits as described above but without the customers needing to own and operate their own equipment. The number of hubs in the network is rapidly growing. At the time of writing, there are 14,300 3D printers accessible within the 3D Hubs network.

Manufacturing and Remanufacturing for Maintenance. The production of the bladed disks ('blisks') used in aero engines has a high environmental impact, with significant material waste. Material input to final component ratios of 4:1 are common using traditional 5-axis milling processes, with some components having a ratio as high as 20:1. In the EU FP7 MERLIN project, Rolls-Royce, Turbomeca, MTU, and Fraunhofer ILT collaborated to address this environmental impact. Early demonstrators showed that AM can be used to manufacture and maintain the blisks and reduce waste with $\sim 60 \%$ material savings and $\sim 30 \%$ time savings [11]. AM can also be used for the in-situ repair of damaged blisks and thereby extend their operational life.

End-of-Life Product Recycling. The Perpetual Plastic Project (PPP) investigated how plastic waste could be used as an input for 3D printing. The materials tested are used in 
everyday products such as cups, bottles, caps and carrier bags; i.e. polylactic acid (PLA), polystyrene (PS), low density polyethylene (LDPE), polyamide (PA) and polypropylene (PP). The project demonstrated the feasibility and relative ease of plastic recycling for 3D printing applications, some more successfully than others.

The bio-polymer PLA can provide a wide range of material properties and thus substitute for different plastics. Through the greater use of PLA and less diversity in the range of plastics consumed, simpler recycling systems may be realised. For example, the Filabot reclaimer grinds plastic goods into granules, which are fed into a Filabot machine to create new 3D printing filament. In addition, PLA has the ability to be recycled with no quality loss when treated by specialised companies (e.g. Plaxica). It can be fed back into the same system and thus enable a closed-loop circulation of material.

Another example is the EKOCYCLE Cube. It uses recycled polyethylene terephtalate (rPET) in its cartridges with $25 \%$ recycled PET content. Higher recycled content is possible but limited by aesthetic requirements. The EKOCYCLE Cube is branded as a lifestyle product that enables creativity in the design and realisation of fashion and music accessories.

\subsection{Barriers and Opportunities}

In this section we discuss the potential benefits of AM to improve the sustainability of products, processes, manufacturing systems and personal lifestyles. We use the different stages shown in Fig. 1 as a guiding structure for the discussion.

Product and Process Design. Traditional manufacturing techniques can be wasteful as they are subtractive. Nature follows an additive process that is more efficient. Components and product assemblies designed for AM mimic nature in the way they are built up. As shown in the GE example, they have fewer parts and more optimised geometries, often unachievable using other manufacturing techniques. Harnessing this freedom in shape and geometry in the design stage achieves novel, more complex (or simpler) structures, including free-form enclosed shapes, channels and lattices.

Just as product design can be improved, so can production process design. As demonstrated by Salcomp, the production process can become more resource efficient by incorporating AM-produced components (e.g. moulds, tooling). This is achieved through a combination of lower energy consumption, and higher quality to reduce rejection rates during the production stage.

In other stages of the product life cycle, AM can improve resource efficiency in manufacturing, improve operational efficiency, functionality and durability in use, and enable reuse, repair and recycling at the end-of-life. Overall, it leads to the decoupling of the total value delivered per unit of resource consumed, as illustrated with the RollsRoyce blisks.

Current barriers to the adoption of AM include how the technology is perceived by designers and the performance limitations of the technology. The first of these barriers stems from the perception held by engineers and designers that AM is only for rapid prototyping and not fit for direct component and product manufacture. Changing the 
way that designers think about AM is a challenge. Without a mindset shift, the full benefits of AM won't be harnessed in the design stage. The second barrier arises from the performance of AM technologies. Current technologies can only produce novel forms; they cannot embed functionality such as microelectronics into components and products. It is likely that a second mindset shift within the design community will be required when AM technologies become more advanced and this functionality can be embedded during the manufacturing process.

Material Input Processing. As the example of Metalysis showed, there is potential to rethink how raw materials are processed to minimise the resources needed to bring them into a usable form as inputs for AM. However, few materials can currently be produced using these novel processing technologies. The processes are immature and the input materials for AM have yet to be standardised. To identify the most resource efficient standards and enable this standardisation to be achieved, further research is required to explore and validate the mechanical and thermal properties of AM technologies and materials.

Regarding the conversion of by-products into products, AM can enable the direct reuse of by-products, such as waste in granulated or powder form, as a material input for production, e.g. Bewell Watches. Using waste as an input to produce personalised products is commonly known as waste upcycling and is advocated by the cradle-tocradle community [12]. However, limitations on the material quality and purity could prevent these products from being recycled when they reach their end-of-life. This is the case with current wood-polymer composites as the technology for material separation and thus recycling does not exist yet.

Make-to-Order Component and Product Manufacturing. AM allows products to be manufactured on demand. This make-to-order model can help eliminate or at least minimise inventory waste, reduce inventory risk with no unsold finished goods, while also improving revenue flow as goods are paid for prior to being manufactured. It allows direct interaction between local consumers and producers, collaborative learning, and user innovation [13]. However, non-linear, localised collaboration between actors with ill-defined roles and responsibilities can result in conflicts and incompatibilities.

Looking at the overall manufacturing system configuration, AM enables a shift from traditional mass production methods and economies of scale to small batch production of customised goods at more affordable prices. Moreover, AM can lead to the reconfiguration of the supply chain as fewer components within product assemblies means that fewer actors, stages and interactions may be needed within it, along with a potential reduction in the environmental impacts of logistics.

As previously described in Section 5.1, the additive nature of AM means that less waste is generated during the production process. While AM can be more energy intensive per unit produced (relative performance), it allows units to be produced to exactly match the demand (make-to-order) and thus reduces the overall amount of resources consumed (absolute performance). In other words, AM can enable dematerialisation and lower energy intensity across the whole system. 
Furthermore, automation is needed if AM is to become more resource efficient. For instance, automated post-processing is needed to achieve desired aesthetic finishes and to eliminate the 'stair stepping' effect arising from the incremental layer-by-layer buildup of material.

Closing the Loop. During repair, maintenance and remanufacturing, a make-to-order model can be applied to minimise inventory waste as spare parts can be produced locally only when needed, with lower energy intensity processes. This is even more the case with modular and upgradable components. Products can be maintained in-situ using AM repair technology, thereby maximising their use and extending their lifespan.

In addition, the availability of AM technologies for repair creates incentives for companies to adopt service-based business models. Such business models have proven highly profitability for companies in the aerospace sector where providing maintenance services allows the manufacturer to satisfy its customers' needs for a high level of flight utilisation.

The AM process has the potential to increase the recovery of value embedded in waste. At the product end-of-life stage, in-situ recycling systems can be linked to AM, diverting material from waste streams into new applications. Closing the loop through recycling can be achieved at various stages and scales in AM. The highest value recovery possible is achieved locally during the manufacturing process when the unused AM material is reclaimed.

Initiatives such as PPP help raise awareness and educate the public about small-scale plastic waste recycling and AM. As AM technology and 3D printed products becoming more attractive, companies are attempting to harness this 'coolness' and enhance their brand identity. This can be seen in approaches such as the EKOCYCLE brand, where an attempt is being made to make recycling more fashionable, overcoming traditional negative perceptions of recycled materials being of lower quality than virgin ones, in particular for certain plastics.

\section{Conclusions}

While AM has yet to dramatically transform industrial systems, there are early signs of how the characteristics of this advanced production process will lead to advances in industrial sustainability. In this paper we have explored the opportunities in the product life cycle for sustainability improvements through the implementation of AM technologies, providing illustrations from practice of the ways in which such improvements are being made.

There are numerous cases of product redesign arising from the application of AM. While the majority of these remain demonstrations that have not entered actual production, high profile examples of AM-based redesign such as the General Electric LEAP engine are bringing about real change and altering industry perceptions of the potential application of AM.

As an emerging technology and industry, there remains significant scope for further adoption and sustainability benefits to be realised. Currently the technology is being 
adopted by user innovators and early adopters and it is far from becoming mainstream practice. While we have provided examples from other phases of the product lifecycle, the number of documented cases is more sparse across these phases. AM can create new business opportunities for reuse, repair, refurbishment and remanufacturing but companies are only just beginning to discover that AM can extend product life cycles and close the loop. This may be best exploited through the adoption of service-based business models and can result in the decoupling of the environmental impacts from the social and economic value created thereby increasing the companies' sustainability performance.

\section{References}

1. Gebler, M., Schoot Uiterkamp, A.J.M., Visser, C.: A global sustainability perspective on 3D printing technologies. Energ. Policy 74 (C), 158-167 (2014)

2. Evans, S., Bergendahl, M.N., Gregory, M., Ryan, C.: Towards a Sustainable Industrial System, with Recommendations for Education, Research, Industry and Policy. University of Cambridge, Institute for Manufacturing, Cambridge (2009)

3. Petrovic, V., Gonzalez, J.V.H., Ferrando, O.J., Gordillo, J.D., Puchades, J.R.B., Grinan, L.P.: Additive layered manufacturing: sectors of industrial application shown through case studies. Int. J. Prod. Res. 49(4), 1061-1079 (2011)

4. Berman, B.: 3-D printing: The new industrial revolution. Bus. Horizons 55, 155-162 (2012)

5. Petrick, I.J., Simpson, T.W.: 3D Printing Disrupts Manufacturing: How Economies of One Create New Rules of Competition. Res. Technol. Manage. 56(6), 12-16 (2013)

6. TSB: Shaping our national competency in additive manufacturing: Technology innovation needs analysis conducted by the Additive Manufacturing Special Interest Group for the Technology Strategy Board. TSB Knowledge Transfer Network Special Interest Group on Additive Manufacturing (2012)

7. SAVING project: http://www.manufacturingthefuture.co.uk/case-studies/

8. General Electric: http://www.gereports.com/post/80701924024/fit-to-print

9. Mellor, I., Grainger, L., Rao, K., Deane, J., Conti, M., Doughty, G., Vaughan, D.: Titanium powder production via the Metalysis process. In: Qian, M., Froes, F.H. (eds.) Titanium Powder Metallurgy: Science, Technology and Applications, pp. 51-67. Butterworth-Heinemann, Oxford (2015)

10. EOS: http://www.eos.info/press/customer_case_studies/salcomp

11. Fraunhofer: http://www.ilt.fraunhofer.de/content/dam/ilt/en/documents/annual_reports/JB11 /JB11_P82.pdf

12. McDonough, W., Braungart, M.: The Upcycle: Beyond Sustainability - Designing for Abundance. North Point Press, New York (2013)

13. de Jong, J.P.J., de Bruijn, E.: Innovation Lessons From 3-D Printing. MIT Sloan Manage. Rev. 54(2), 42-52 (2013) 